\title{
Unusual presentations, management and outcomes of gastric stromal tumors: a single-center case series
}

\author{
Alan Askaria, Rory Brittain ${ }^{a}$, Marwa Hilmi ${ }^{b}$, Wasim Hajuthman ${ }^{a}$, Ahmed Al-Bahrania \\ West Hertfordshire Hospitals NHS Trust, Watford; General Practitioner, Lodge Surgery, St Albans, UK
}

Abstract

\section{Introduction}

Gastrointestinal (GI) stromal tumors (GIST) are rare tumors with an incidence of 10-20 cases per one million of the population; the highest rates are in China and northern Norway [1-5]. They arise from muscle or connective tissues of the GI tract and can occur at any GI site, but tend to

${ }^{a}$ Department of General Surgery, West Hertfordshire Hospitals NHS Trust, Vicarage Road, Watford (Alan Askari, Rory Brittain, Wasim Hajuthman, Ahmed Al-Bahrani); 'General Practitioner, Lodge Surgery, St Albans (Marwa Hilmi), United Kingdom

Conflict of Interest: None

Correspondence to: Alan Askari, Department of General Surgery, West Hertfordshire Hospitals NHS Trust, Vicarage Road, Watford, WD18 0UB, United Kingdom, e-mail: alan.askari@nhs.net

Received 6 March 2020; accepted 9 July 2020; published online 2 October 2020

DOI: https://doi.org/10.20524/aog.2020.0540 occur most frequently in the stomach and small bowel [6]. Whilst the majority are benign, they have potential for malignancy, particularly non-gastric GISTs [7]. There is considerably variability in the tendency for these tumors to turn malignant and their histological differentiation is equally heterogeneous.

Clinically, patients can present with a variety of symptoms, depending on the size and the site of the tumor. Larger tumors may exert a mass effect and cause abdominal pain, or bleeding in the GI tract, although a significant proportion may be entirely asymptomatic and their discovery incidental. Surgical resection remains the mainstay treatment of GISTs, although the use of other approaches (notably endoscopic) has been increasing [8], despite reports of potential tumor rupture during the procedure [9]. Laparoscopic resection has become increasingly attractive recently, and its short- and long-term outcomes appear favorable [10]. A recent metaanalysis deemed laparoscopy to have potentially favorable outcomes over endoscopic treatment of GIST [11]. The aim of this study was to report our short and long-term outcomes 
from a large case series of patients with gastric GIST and to highlight unusual signs and symptoms which patients may present.

\section{Patients and methods}

A single-institution dataset prospectively maintained was used for this study. All consecutive patients undergoing gastric GIST resection between January 2011 and June 2018 were included in the study. Patient demographics, comorbidities, surgical access (laparoscopic/open), length of stay and complications were all recorded.

\section{Diagnosis and tumor assessment}

Our institution receives referrals from general practice, other hospitals (via a tertiary referral system), as well as via the emergency department at the hospital itself. Upon diagnosis, patients would go through our multidisciplinary team meeting, comprising upper GI surgeons, gastroenterologists, oncologists and radiologists. All patients underwent esophagogastroduodenoscopy and computed tomography (CT) to assess the characteristics of the GIST. In a selection of patients who required further diagnostic clarity or evaluation of the tumor and obtaining pathological sample, endoscopic ultrasound was used. The risk of progression was calculated using modified National Institute of Health risk classification criteria, as set out by Joensuu et al and published in 2008 [12].

\section{Treatment and operative approach}

The multidisciplinary team made recommendations for treatment in line with the patient's wishes, based on patient comorbidity, tumor location, size, risk of malignancy, and previous surgical history. Laparoscopy was the preferred surgical approach in all patients with tumors up to $5 \mathrm{~cm}$ in size. In patients with tumors between 5 and $10 \mathrm{~cm}$, laparoscopy was attempted but there was a low threshold for conversion to open. In patients who had undergone multiple previous surgeries or had large tumors $(>10 \mathrm{~cm})$, an open method was adopted. The surgeon's experience could play a role in the surgical approach. In case of tumors not amenable to resection because of their large size $(10 \mathrm{~cm}+)$ or involvement of surrounding structures that mandated multi-organ resection, imatinib was given as neoadjuvant therapy.

\section{Statistical analysis}

Scale variables were reported as medians with accompanying interquartile range (IQR), groups were compared using Pearson chi-squared analysis and a P-value of $<0.05$ was considered statistically significant. Statistical analysis was carried out using the Statistical Package for the Social Sciences (SPSS) IBM version 23 .

\section{Results}

\section{Population characteristics}

A total of 69 patients (Table 1) underwent gastric GIST resection, of whom $56.5 \%(n=39 / 69)$ were male; the patients' median age was 68 years (IQR 60-76 years). The median tumor size was $5.0 \mathrm{~cm}$ (IQR $4.0-7.2 \mathrm{~cm}$ ). The majority of the patients had an American Society of Anesthesiology grade II ( $n=47 / 69$, $68.1 \%)$, a smaller proportion $(17.4 \%, n=12 / 69)$ were grade III and the remainder $(14.5 \%, \mathrm{n}=10 / 69)$ were grade I. In 43 patients $(62.3 \%)$ the GIST was in the gastric body, whilst in 19 patients $(27.5 \%)$ and 6 patients $(8.7 \%)$ it was in the gastric fundus or antrum, respectively. In one patient the tumor was located in the pylorus. Overall, $72.5 \%(n=50 / 69)$ had a GIST

Table 1 Patient demographics, tumor characteristics and outcomes

\begin{tabular}{|c|c|c|c|}
\hline \multicolumn{2}{|l|}{ Characteristics } & \multirow{2}{*}{$\begin{array}{c}n \\
39\end{array}$} & \multirow{2}{*}{$\begin{array}{c}\% \\
56.5 \%\end{array}$} \\
\hline Sex & Male & & \\
\hline & Female & 30 & $43.5 \%$ \\
\hline \multirow[t]{4}{*}{ Age } & $<60$ years & 17 & $24.6 \%$ \\
\hline & $60-69$ years & 20 & $29.0 \%$ \\
\hline & $70-79$ years & 19 & $27.5 \%$ \\
\hline & $80+$ years & 13 & $18.8 \%$ \\
\hline \multirow[t]{4}{*}{ Tumor site } & Gastric antrum & 6 & $8.7 \%$ \\
\hline & Gastric body & 43 & $62.3 \%$ \\
\hline & Gastric fundus & 19 & $27.5 \%$ \\
\hline & Gastric pylorus & 1 & $1.4 \%$ \\
\hline \multirow{3}{*}{$\begin{array}{l}\text { Risk of } \\
\text { progression }\end{array}$} & Low & 50 & $72.5 \%$ \\
\hline & Intermediate & 9 & $13.0 \%$ \\
\hline & High & 10 & $14.5 \%$ \\
\hline \multirow[t]{3}{*}{ Treatment } & Surgery & 63 & $91.3 \%$ \\
\hline & Surgery \& adjuvant chemotherapy & 1 & $1.4 \%$ \\
\hline & $\begin{array}{l}\text { Surgery \& neoadjuvant } \\
\text { chemotherapy }\end{array}$ & 5 & $7.2 \%$ \\
\hline \multirow[t]{2}{*}{ Surgery type } & Gastrectomy & 12 & $17.4 \%$ \\
\hline & GIST excision & 57 & $82.6 \%$ \\
\hline \multirow[t]{3}{*}{ Surgical access } & Laparoscopic & 54 & $78.3 \%$ \\
\hline & Open & 12 & $17.4 \%$ \\
\hline & Converted to open & 3 & $4.3 \%$ \\
\hline \multirow{2}{*}{$\begin{array}{l}\text { Postoperative } \\
\text { complication }\end{array}$} & Yes & 6 & $8.7 \%$ \\
\hline & No & 63 & $91.3 \%$ \\
\hline \multirow[t]{2}{*}{ Mortality status } & Alive & 62 & $89.9 \%$ \\
\hline & Deceased & 7 & $10.1 \%$ \\
\hline
\end{tabular}

GIST, gastrointestinal tumor 
histopathologically graded as low risk of progression, whilst $14.5 \%(n=10 / 69)$ had a high-risk tumor.

\section{Surgical outcomes}

The majority of the patients $(78.3 \%, \mathrm{n}=54 / 69)$ underwent a laparoscopic resection, whilst $17.4 \%(n=12 / 69)$ had an open procedure. In $4.3 \%(n=3 / 69)$ of cases, a laparoscopic procedure was converted to open. All 3 of these latter patients had had previous major abdominal surgery. There was a higher proportion of patients undergoing gastrectomy in the open group $(66.7 \%)$ compared with the laparoscopic group ( $8.9 \%, \mathrm{P}<0.001$, Table 2 ). Patients in the open group had a significantly higher proportion of patients with high-risk tumors (50\%) compared with the laparoscopic group $(3.7 \%, \mathrm{P}=0.001)$. Most patients only required a GIST excision $(82.6 \%, n=57 / 69)$, whilst $17.4 \%(n=12 / 69)$ required a gastrectomy ( 1 of whom had a Merendino type resection). The median length of stay was 7 days (IQR 4-10 days) across the entire population and was significantly lower in the laparoscopy group (6 days, IQR 5-10) compared with the open group (9 days, IQR 7-13, P=0.003). A total of 6 complications $(8.7 \%)$ occurred and complication rates by surgical approach were similar (7.4\% in the laparoscopy group and $8.3 \%$ in the open group, $\mathrm{P}=0.882$ ). These 6 complications included 2 patients who experienced GI bleeding/melena, probably due to the gastrojejunal anastomosis site, which resolved without any intervention. Two patients developed postoperative pneumonia and 1 elderly female patient developed a urinary tract infection postoperatively; all were treated with antibiotics. One patient developed a small subphrenic collection (also treated with antibiotics) and 1 patient developed a pyloric stricture as demonstrated on oral contrast study; however, the patient had mild symptoms and did not require intervention. There were no returns to theater from any of the above complications.

\section{Long-term outcome}

None of the patients were lost to follow up and at a median of 53 months' follow up (IQR 26-75 months), 89.9\% ( $n=62 / 69$ ) of the population were alive. One patient who underwent a

Table 2 Demographics and outcomes according to surgical access

\begin{tabular}{|c|c|c|c|c|c|c|c|c|}
\hline \multirow[t]{2}{*}{ Characteristics } & & \multicolumn{2}{|c|}{ Laparoscopic } & \multicolumn{2}{|c|}{ Open } & \multicolumn{2}{|c|}{ Converted to open } & \multirow[t]{2}{*}{ P-value } \\
\hline & & $\mathrm{n}$ & $\%$ & $\mathrm{n}$ & $\%$ & $\mathrm{n}$ & $\%$ & \\
\hline \multirow[t]{2}{*}{ Sex } & Male & 30 & $55.6 \%$ & 9 & $75.0 \%$ & 0 & $0.0 \%$ & 0.061 \\
\hline & Female & 24 & $44.4 \%$ & 3 & $25.0 \%$ & 3 & $100.0 \%$ & \\
\hline \multirow[t]{4}{*}{ Age } & $<60$ years & 11 & $20.4 \%$ & 6 & $50.0 \%$ & 0 & $0.0 \%$ & 0.247 \\
\hline & $60-69$ years & 17 & $31.5 \%$ & 2 & $16.7 \%$ & 1 & $33.3 \%$ & \\
\hline & $70-79$ years & 15 & $27.8 \%$ & 2 & $16.7 \%$ & 2 & $66.7 \%$ & \\
\hline & $80+$ years & 11 & $20.4 \%$ & 2 & $16.7 \%$ & 0 & $0.0 \%$ & \\
\hline \multirow[t]{4}{*}{ Tumor site } & Gastric antrum & 4 & $7.4 \%$ & 2 & $16.7 \%$ & 0 & $0.0 \%$ & 0.197 \\
\hline & Gastric body & 36 & $66.7 \%$ & 6 & $50.0 \%$ & 1 & $33.3 \%$ & \\
\hline & Gastric fundus & 14 & $25.9 \%$ & 3 & $25.0 \%$ & 2 & $66.7 \%$ & \\
\hline & Gastric pylorus & 0 & $0.0 \%$ & 1 & $8.3 \%$ & 0 & $0.0 \%$ & \\
\hline \multirow[t]{3}{*}{ Risk of progression } & Low & 45 & $83.3 \%$ & 4 & $33.3 \%$ & 1 & $33.3 \%$ & 0.001 \\
\hline & Intermediate & 7 & $13.0 \%$ & 2 & $16.7 \%$ & 0 & $0.0 \%$ & \\
\hline & High & 2 & $3.7 \%$ & 6 & $50.0 \%$ & 2 & $66.7 \%$ & \\
\hline \multirow[t]{3}{*}{ Treatment } & Surgery & 52 & $96.3 \%$ & 8 & $66.7 \%$ & 3 & $100.0 \%$ & 0.331 \\
\hline & Surgery \& adjuvant chemotherapy & 1 & $1.9 \%$ & 0 & $0.0 \%$ & 0 & $0.0 \%$ & \\
\hline & Surgery \& neo-adjuvant chemotherapy & 1 & $1.9 \%$ & 4 & $33.3 \%$ & 0 & $0.0 \%$ & \\
\hline \multirow[t]{2}{*}{ Surgery type } & Gastrectomy & 3 & $8.9 \%$ & 8 & $66.7 \%$ & 1 & $33.3 \%$ & $<0.001$ \\
\hline & GIST excision & 51 & $91.1 \%$ & 4 & $33.3 \%$ & 2 & $66.7 \%$ & \\
\hline \multirow[t]{2}{*}{ Complication } & Yes & 4 & $7.4 \%$ & 1 & $8.3 \%$ & 1 & $33.3 \%$ & 0.300 \\
\hline & No & 50 & $92.6 \%$ & 11 & $91.7 \%$ & 2 & $66.7 \%$ & \\
\hline \multirow[t]{2}{*}{ Mortality status } & Alive & 53 & $98.1 \%$ & 7 & $58.3 \%$ & 2 & $66.7 \%$ & $<0.001$ \\
\hline & Deceased & 1 & $1.9 \%$ & 5 & $41.7 \%$ & 1 & $33.3 \%$ & \\
\hline
\end{tabular}


laparoscopic resection had a recurrence (1.4\%), despite having an $\mathrm{R} 0$ resection and a tumor grade deemed to have low risk for recurrence (1.9\% risk of progression). Overall survival analyses were undertaken and all-cause mortality was considered. Survival analysis demonstrated an overall mean survival of 92.7 months (95\% confidence Interval [CI] 86.3-99.2). This was significantly better in the laparoscopy group, who had a mean survival of 100.4 months (95\%CI 97.2-103.6), compared with 55.1 months (95\%CI 41.2-68.9) in the open group and 59.3 months (95\%CI 11.9-106.8) in the laparoscopy converted to open group $(\mathrm{P}<0.001$, Fig. 1$)$. Patients in the open group had a greater proportion of patients who had high-risk tumors $(50 \%, n=6 / 12)$ compared with the laparoscopic group $(3.7 \%$, $\mathrm{n}=2 / 54, \mathrm{P}=0.001$ ), and mortality was also significantly higher in the open group compared with the laparoscopic group ( $41.7 \%$ vs. $1.9 \%, \mathrm{P}<0.001$ ), reflecting the advanced tumors presented by patients in the open group.

\section{Uncommon presentation}

Three of the patients presented with unusual or uncommon findings. One female patient presented with dysphagia and epigastric pain and a mass was identified in the mediastinum on CT. Endoscopic examination of the esophagus and stomach, however, was normal. She underwent surgery and an entirely extraluminal GIST at the gastroesophageal junction was resected. The patient recovered well without any complications. Another young female patient presented with major hemorrhage requiring multiple blood transfusions and urgent laparotomy. At laparotomy, an exophytic GIST on the greater curvature was found to be actively bleeding intraperitoneally. The third patient was a 51-year-old female who presented as an emergency with sudden-onset epigastric pain. Subsequent CT imaging demonstrated a perforated mass in the gastric antrum that required immediate laparotomy and distal gastrectomy. These 3 cases highlight some of the unusual ways which these tumors can present, ranging from unseen masses on esophagogastroduodenoscopy, causing extrinsic compression of the esophagus and dysphagia, to catastrophic bleeding and perforation.

\section{Discussion}

The main finding of this single-institution case series was that gastric GIST excision surgery was associated with a low risk of complications and good long-term survival. Laparoscopic resections were safe for selected gastric GISTs in favorable anatomic locations (greater curvature or anterior wall of the stomach), for smaller size GISTs, and when performed by surgeons with appropriate experience. They were associated with a lower risk of complications and equivalent long-term outcomes compared to open surgery. Our findings are similar to those reported by others. In one of the largest studies, involving 1057 patients across Korea and Japan, the authors found low rates of 30 -day mortality $(0.2 \%)$ and postoperative complications $(8.1 \%)$, and only a 5\% 5-year recurrence rate [13].

Much like the use of laparoscopy in other GI tumors (notably colon), the use of a minimally invasive approach was initially met with suspicion. Concerns over marginal clearance and non-disruption of the tumor itself were widespread at first. However, as laparoscopic resection for a variety of tumors, including esophageal, gastric, liver, kidney, colon and even pancreatic, have become commonplace, these concerns have largely been demonstrated to be unfounded. In our case series, the majority of patients $(78.9 \%, \mathrm{n}=56)$ underwent a laparoscopic resection; all had clear margins, with no recurrence at a median 4 years of follow up. The first laparoscopic excision of a GIST, carried out in 1992, was incidental [14]. Since then, a number of case series and observational studies have demonstrated that laparoscopic surgery is not only feasible and safe in GIST

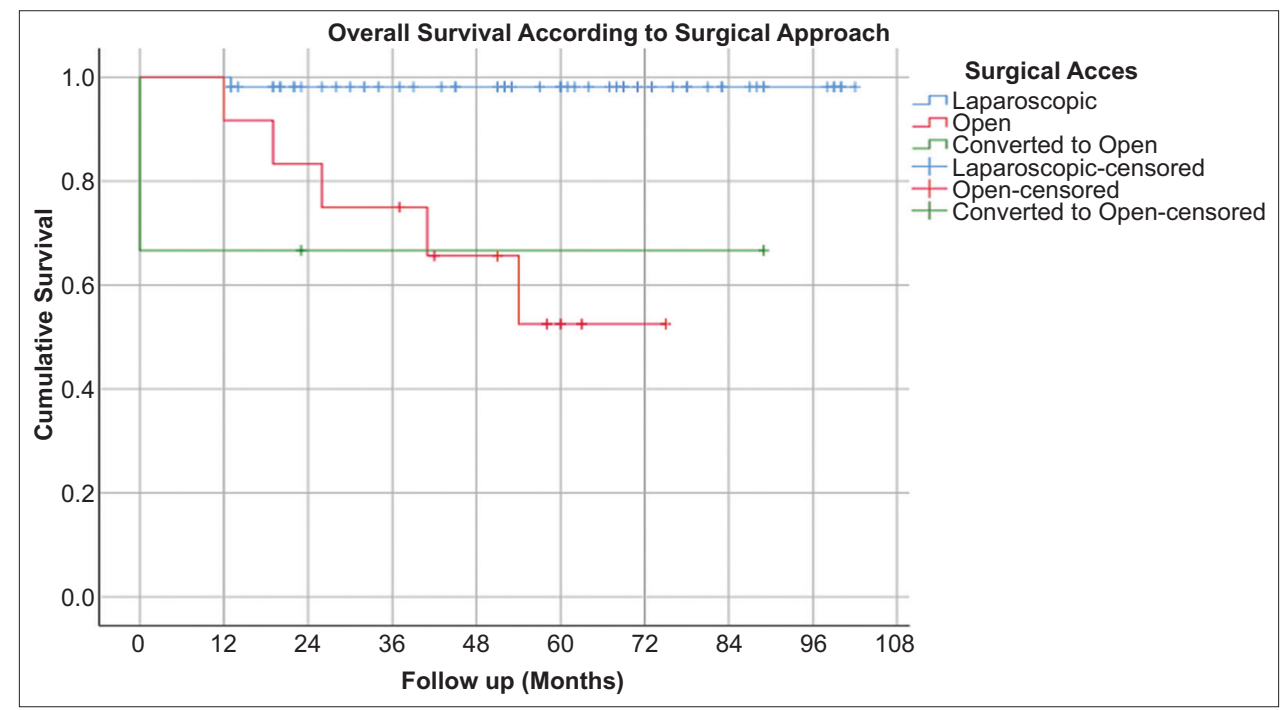

Figure 1 Survival analysis comparing the populations in the different surgical access groups 
excision, but is also associated with a shorter length of stay, lower risk of postoperative ileus and quicker return to bowel function, and has long-term oncological outcomes equivalent to those of open surgery [15-20]. As yet, however, there has been no definitive trial data for laparoscopic vs. open surgery in the surgical management of GIST. In our study, patients in the laparoscopic group fared better in terms of long-term survival; however, this is most probably because the laparoscopic group had a lower rate of aggressive/advanced tumors.

Therefore, it would be erroneous to presume laparoscopic superiority over open surgery in terms of oncological outcome. However, the findings do demonstrate that it is at least not inferior to open surgery, and that laparoscopic surgery can provide clear margins and equivalent long-term results, with the benefits of decreased tissue trauma and length of stay perioperatively. Our data also demonstrated that the majority of patients could have a laparoscopic excision with a relatively low risk of having to convert to open. In this study, the conversion rate from laparoscopic to open surgery was $4.3 \%$ (3 patients), in keeping with the published literature, which quotes a conversion rate of around 6\% [21]. Other concerns around laparoscopic use center on port site metastases. Resected specimens should be retrieved from the abdomen in a plastic bag to avoid rupture and intraperitoneal spillage. Whilst there are case reports of port site seeding from sampling GISTs laparoscopically [22], this is extremely rare and sampling itself has largely become a thing of the past, as modern imaging modalities such as CT or endoscopic ultrasonography are usually adequate for establishing a diagnosis without the need for sampling. In cases where there is diagnostic uncertainty, or where quantification of the risk of malignancy is required, preoperative biopsies can be useful. Furthermore, preoperative biopsies may be helpful in confirming metastatic disease and in certain institutions a preoperative biopsy is mandatory prior to the initiation of definitive treatment, although there are concerns about potential tumor seeding via a transabdominal biopsy tract [23].

A variety of factors can affect the decision to undertake laparoscopic resection in GIST patients. Previous abdominal surgery, tumor size and location, resource/equipment availability, as well as the surgeon's experience or preference, all have a role to play in the decision whether to undertake laparoscopic or open surgery. Although rare, GISTs can sometimes present as emergencies, usually with substantial intraabdominal hemorrhage and bowel obstruction [24,25]. The concern in undertaking GIST resection in the non-elective setting is potential breach of the tumor capsule, which has been associated with a significant risk of recurrence and metastases. In our study, 3 patients presented in an unusual way. One female patient presented with catastrophic internal hemorrhage requiring multiple blood transfusions and an urgent life-saving laparotomy. Another female patient had a complete exophytic tumor not detected on gastroscopy. There are several reports of atypical presentation of GISTs affecting a variety of organs, including the liver, prostate, ovaries, uterus, omentum and rectum, amongst others. Such unusual and potentially lifethreatening presentations are rare; however, a high index of suspicion is required and timely surgical treatment of known GISTs is essential to avoid their presentation acutely, as this invariably results in a poorer outcome for the patient.

Overall survival from GISTs is variable, given their vastly heterogeneous nature. Unsurprisingly, much like other tumors, survival seems to be at least in part dependent on tumor DNA, mitotic count and tumor differentiation, rather than on tumor size alone [26]. Tumors that have a high mitotic index, i.e., above 10/50 high-power field, and those greater than $5 \mathrm{~cm}$ in size have been demonstrated to adversely impact survival [27]. This discrepancy in the risk of progression between the open and laparoscopic groups is the likely explanation for the low recurrence and mortality rate in the laparoscopic group compared to the open group. One of the aims of this study was to demonstrate whether the laparoscopic approach is safe in the short and long term and is comparable to the open approach in terms of recurrence and subsequent survival, rather than to demonstrate superiority. Undoubtedly, open surgery continues to have a major role. Other negative prognostic indicators include advanced age, non-gastric tumors and incomplete resection of tumor (R1) or tumor rupture. Perhaps one of the biggest factors affecting survival is tumor rupture. The Oslo criteria set out a range of definitions of what constitutes tumor rupture. These include both natural and iatrogenic causes of rupture. Spontaneous rupture of tumor causing hemorrhage and peritonitis, GI organ perforation through the tumor, and blood-stained ascites are concerning features of tumor rupture. Similarly, iatrogenic causes can arise during laparoscopic surgery itself, when the tumor capsule is breached and piecemeal resection occurs. Importantly, R1 resections, tumor spillage into GI lumen, microscopic tumor penetration into the peritoneum and uncomplicated transperitoneal needle biopsy without complication do not constitute tumor rupture. Incisional biopsies, however, would qualify as tumor rupture and must therefore not be attempted in suspected GISTs [28].

A factor that positively affects survival is the administration of postoperative imatinib (Glivec) to patients with high-risk tumors, although no survival benefit was noted in patients with intermediate risk [27]. The duration of imatinib therapy is also important. An analysis of trial data of 400 patients with GIST has demonstrated a significant survival benefit in patients who have 3 years of imatinib as opposed to 1 year [29]. Imatinib has also been demonstrated to improve quality of life in GIST patients at high risk of recurrence, although the financial cost of long-term therapy may be prohibitive in certain parts of the world [30]. The efficacy of imatinib has led to it being recommended as essential adjuvant therapy in patients with GISTs that are at high-risk (and even arguably intermediate risk) of recurrence and those patients who have had a spontaneous or iatrogenic tumor rupture. However, despite the recommendations put forward by the US based National Comprehensive Cancer Network and the European Society for Medical Oncology, advocating the use of imatinib in high-risk and even intermediate risk GISTs, many institutions are not consistently offering high-risk patients adjuvant imatinib and the reasons for this are not entirely clear [31]. In our case series, 5 patients had neoadjuvant imatinib therapy and only 1 had adjuvant therapy, despite 10 patients being deemed high-risk 
for disease progression. A concerted effort is required to identify patients with a high risk of disease progression and in appropriate circumstances offer imatinib therapy. The case for routinely administering imatinib to intermediate risk patients is less clear, with no convincing evidence to justify its use.

The main limitations of our study were that it was a singlecenter, retrospective case series, prone to certain inherent biases. Our sample size was small, making it difficult to draw definitive conclusions. Our population may also be different to that of other hospitals and our results not entirely applicable to populations of other regions of the world. Moreover, although in our series survival appeared to be better in the laparoscopic group, this was almost certainly due to a difference in the case mix of the patients undergoing open and laparoscopic surgery. Tumors in unfavorable anatomic locations (lesser curvature, posterior gastric wall or in proximity to cardia or pylorus) are more likely to have an open resection rather than a laparoscopic one. Therefore, there is still a definite role for open surgery in patients with excessively large tumors, particularly in the nonelective setting or in patients who have had multiple previous abdominal surgeries for other pathology, making laparoscopy challenging or unsafe. Additionally, in less common cases with pathologically enlarged regional lymph nodes (succinate dehydrogenase-deficient GIST), where a lymphadenectomy, is indicated open surgery constitutes a more realistic approach.

In conclusion, this single-center case series has demonstrated that, although most gastric GISTs are incidental findings or are diagnosed in a non-emergency setting, some patients can present in unusual ways. Large tumor sizes and patients at high risk of progression are important considerations when deciding upon the surgical approach. In appropriately selected patients, laparoscopy is safe, has good perioperative outcomes and is equivalent to open surgery in terms of oncological outcome. The use of adjuvant imatinib should be discussed with all patients who have GISTs deemed to be high-risk.

\section{Summary Box}

\section{What is already known:}

- Gastrointestinal tumors (GISTs) are tumors with a potential for malignancy and can present in unusual ways

- Overall survival post-surgical resection is very good provided an R0 resection is achieved

\section{What the new findings are:}

- Laparoscopic surgery is a safe alternative to open surgery in appropriately selected patients and entails a shorter length of stay

- The $\mathrm{R} 1$ resection rate is low, irrespective of the surgical approach taken

- Survival and complication rates are comparable between open and laparoscopic surgery

\section{References}

1. Eisenberg BL, Trent JC. Adjuvant and neoadjuvant imatinib therapy: current role in the management of gastrointestinal stromal tumors. Int J Cancer 2011;129:2533-2542.

2. Miettinen M, Lasota J. Histopathology of gastrointestinal stromal tumor. J Surg Oncol 2011;104:865-873.

3. Miettinen M, Lasota J. Gastrointestinal stromal tumors-definition, clinical, histological, immunohistochemical, and molecular genetic features and differential diagnosis. Virchows Arch 2001;438:1-12.

4. Chen $\mathrm{YH}$, Liu $\mathrm{KH}$, Yeh $\mathrm{CN}$, et al. Laparoscopic resection of gastrointestinal stromal tumors: safe, efficient, and comparable oncologic outcomes. J Laparoendosc Adv Surg Tech A 2012;22:758-763.

5. Søreide K, Sandvik OM, Søreide JA, Giljaca V, Jureckova A, Bulusu VR. Global epidemiology of gastrointestinal stromal tumours (GIST): A systematic review of population-based cohort studies. Cancer Epidemiol 2016;40:39-46.

6. Miettinen M, Lasota J. Gastrointestinal stromal tumors: pathology and prognosis at different sites. Semin Diagn Pathol 2006;23:70-83.

7. Nishida T, Goto O, Raut CP, Yahagi N. Diagnostic and treatment strategy for small gastrointestinal stromal tumors. Cancer 2016;122:3110-3118.

8. Dhaliwal AJ, Mashiana HS, Singh AP, Sayles H, Bhat I, Singh S. Sa1935 efficacy and safety of endoscopic resection for the gastrointestinal stromal tumors: a systematic review and metaanalysis. Gastrointest Endosc 2018;87:AB256-AB257.

9. Song S, Ren W, Wang Y, et al. Tumor rupture of gastric gastrointestinal stromal tumors during endoscopic resection: a risk factor for peritoneal metastasis? Endosc Int Open 2018;6:E950-E956.

10. Chen K, Zhou Y-C, Mou Y-P, Xu X-W, Jin W-W, Ajoodhea H. Systematic review and meta-analysis of safety and efficacy of laparoscopic resection for gastrointestinal stromal tumors of the stomach. Surg Endosc 2015;29:355-367.

11. Dong X, Chen W, Cui Z, et al. Laparoscopic resection is better than endoscopic dissection for gastric gastrointestinal stromal tumor between 2 and $5 \mathrm{~cm}$ in size: a case-matched study in a gastrointestinal center. Surg Endosc 2019 Dec 2 [Online ahead of print]. doi: 10.1007/s00464-019-07251-6

12. Joensuu H. Risk stratification of patients diagnosed with gastrointestinal stromal tumor. Hum Pathol 2008;39:1411-1419.

13. Kim MC, Yook JH, Yang HK, et al. Long-term surgical outcome of 1057 gastric GISTs according to 7th UICC/AJCC TNM system: multicenter observational study from Korea and Japan. Medicine (Baltimore) 2015;94:e1526.

14. Lukaszczyk JJ, Preletz RJ. Laparoscopic resection of benign stromal tumor of the stomach. J Laparoendosc Surg 1992;2:331-334.

15. Wan P, Li C, Yan M, Yan C, Zhu ZG. Laparoscopy-assisted versus open surgery for gastrointestinal stromal tumors of jejunum and ileum: perioperative outcomes and long-term follow-up experience. Am Surg 2012;78:1399-1404.

16. Ihn K, Hyung WJ, Kim HI, et al. Treatment results of small intestinal gastrointestinal stromal tumors less than $10 \mathrm{~cm}$ in diameter: a comparison between laparoscopy and open surgery. $J$ Gastric Cancer 2012;12:243-248.

17. Loureiro MP, Almeida RA, Claus CM, et al. Laparoscopic resection of gastrointestinal stromal tumors (GIST). Arq Bras Cir Dig 2016;29:1-4.

18. Qiu G, Wang J, Che X, et al. Laparoscopic versus open resection of gastric gastrointestinal stromal tumors larger than $5 \mathrm{~cm}$ : a singlecenter, retrospective study. Surg Innov 2017;24:582-589.

19. Dressler JA, Palazzo F, Berger AC, et al. Long-term functional outcomes of laparoscopic resection for gastric gastrointestinal stromal tumors. Surg Endosc 2016;30:1592-1598.

20. Nakanishi R, Takahashi I, Kajiwara Y, et al. Laparoscopic and open resection of gastrointestinal stromal tumors of the stomach. 
Fukuoka Igaku Zasshi 2015;106:240-246.

21. Kim JJ, Lim JY, Nguyen SQ. Laparoscopic resection of gastrointestinal stromal tumors: does laparoscopic surgery provide an adequate oncologic resection? World J Gastrointest Endosc 2017;9:448-455.

22. Davies AR, Ahmed W, Purkiss SF. Port site metastasis following diagnostic laparoscopy for a malignant gastro-intestinal stromal tumour. World J Surg Oncol 2008;6:55.

23. Eriksson M, Reichardt P, Sundby H K, et al. Needle biopsy through the abdominal wall for the diagnosis of gastrointestinal stromal tumour - does it increase the risk for tumour cell seeding and recurrence? Eur J Cancer 2016;59:128-133.

24. Khuri S, Gilshtein H, Darawshy AA, Bahouth H, Kluger Y. Primary small bowel GIST presenting as a life-threatening emergency: a report of two cases. Case Rep Surg 2017;2017:1814254.

25. Sorour MA, Kassem MI, Ghazal AE-HA, El-Riwini MT, Abu N A. Gastrointestinal stromal tumors (GIST) related emergencies. Int $J$ Surg 2014;12:269-280.

26. el-Naggar AK, Ro JY, McLemore D, Garnsey L, Ordonez N, MacKay B. Gastrointestinal stromal tumors: DNA flow-cytometric study of 58 patients with at least five years of follow-up. Mod Pathol 1989;2:511-515.

27. Liu X, Qiu H, Zhang P, et al; China Gastrointestinal Stromal Tumor Study Group (CN-GIST). Prognostic factors of primary gastrointestinal stromal tumors: a cohort study based on highvolume centers. Chin J Cancer Res 2018;30:61-71.

28. Nishida T, Hølmebakk T, Raut CP, Rutkowski P. Defining tumor rupture in gastrointestinal stromal tumor. Ann Surg Oncol 2019;26:1669-1675.

29. Joensuu H, Eriksson M, Sundby Hall K, et al. Adjuvant imatinib for high-risk GI stromal tumor: analysis of a randomized trial. J Clin Oncol 2016;34:244-250.

30. Bussabawalai T, Thiboonboon K, Teerawattananon Y. Cost-utility analysis of adjuvant imatinib treatment in patients with high risk of recurrence after gastrointestinal stromal tumour (GIST) resection in Thailand. Cost Eff Resour Alloc 2019;17:1. doi: 10.1186/s12962018-0169-9

31. Bischof DA, Dodson R, Jimenez MC, et al. Adherence to guidelines for adjuvant imatinib therapy for GIST: a multi-institutional analysis. J Gastrointest Surg 2015;19:1022-1028. 